Karin Priem, Université du Luxembourg

\title{
L’album de David Seymour sur la lutte contre l'analphabétisme en Calabre en tant qu'outil de médiatisation : traces matérielles de la rédaction et de la narration visuelle
}

\section{Introduction}

La numérisation des archives de l'UNESCO a permis de mettre au jour un album photographique (contenant des planches-contact, des commentaires et des légendes) qui a pour sujet le contexte social, économique, politique et culturel de l'analphabétisme en Italie, réalisé pour l'UNESCO en 1950 par le cofondateur de Magnum, David Seymour. ${ }^{1}$ À l'époque, Seymour était déjà célèbre pour ses reportages sur la guerre civile espagnole et sur les enfants d'Europe au lendemain de la Seconde Guerre mondiale. $^{2}$

\begin{abstract}
1 L'album est accessible en ligne à https://digital.archives.unesco.org/en/collection/photos/detail/ 16ea6d91-6ccb-a22f-6211-4bdd992291cb/media/2544d484-67f4-18c3-5e62-ad38466c02bc ; archives de l'UNESCO réf.: AG 12, Photo Albums, 1 - [Seymour - Calabre], 1950. Sur le contexte archivistique plus large de l'album de David Seymour, voir l'essai de Giovanna Hendel dans ce volume. Sur l'histoire de l'éducation et l'histoire visuelle de la lutte contre l'analphabétisme dans le sud de l'Italie, voir aussi Roberto Sani : Education, School and Cultural Processes in Contemporary Italy. Macerata: EUM - Edizioni Università di Macerata 2018 ; Juri Meda : «Invisible Schools » : The Public Image of Rural Schools in Southern Italy in Photographic Inquiries and Photo-Reportages (1925-55). In : Historia y Memoria de la Educación 8 (2018), p. 347-396.
\end{abstract}

2 Voir Carole Naggar : David Seymour. Arles : Actes Sud 2011 ; Carole Naggar : Chim: Children of War. New York : Umbrage 2013 ; Carole Naggar : A Second Look : Chim's Children of War. In : Time (16 janvier 2013), http://time.com/3796013/a-second-look-chims-children-of-war/\#1; Carole Naggar : David Seymour: Vies de Chim. Biarritz : Contrejour 2014 ; Carole Naggar : Unravelling a 70-Year-Old Photographic Mystery. In : Time Lightbox (12 avril 2017), http://time.com/4735368/tereska-davidchim-seymour/ ; Carole Naggar : Tereska et son photographe. Paris: The Eyes 2019 ; Karin Priem/ Frederik Herman : Photographs as Living and Entangled Objects : David Seymour's Children of Europe. In : Tim Allender/Inés Dussel/Ian Grosvenor/Karin Priem (éds.) : The Visual in Educational History: Transnational Reflections on the Practice of History in the Digital Age. Berlin : De Gruyter, à paraitre ; voir aussi les publications de l'UNESCO : L'entr'aide internationale par l'image. In : Le Courrier de l'UNESCO 1, $\mathrm{n}^{\circ} 4$ (1948), p. 3 ; Les Communautés d'enfants groupées en fédération. In : Le Courrier de l'UNESCO 1, $\mathrm{n}^{\circ} 7$ (1948), p. 1-2 ; Les Enfants qui n'ont plus de maison : complainte. In : Le Courrier de l'UNESCO 2, nº 1 (1949), p. 5 ; Un immense malheur : les enfants d'Europe. In : Le

Je voudrais remercier Giovanna Hendel et Carole Naggar qui ont généreusement partagé leurs connaissances et leurs expertises avec moi. Leur contribution et nos discussions communes m'ont aidée à élaborer et à développer cet essai.

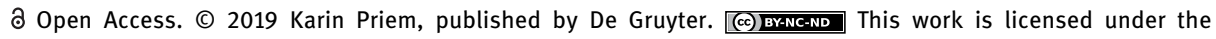
Creative Commons Attribution-NonCommercial-NoDerivatives 4.0 License.

https://doi.org/10.1515/9783110655599-016 
En mars 1950, David Seymour, très inspiré par l'écrivain et peintre italien Carlo Levi, entreprit un reportage ambitieux sur l'analphabétisme, alors un problème majeur dans le sud de l'Italie. ${ }^{3}$ Pour cette commande de l'UNESCO, il se rendit dans plusieurs villages isolés de la région de Calabre : Roggiano Gravina, Bagaladi, Saucci, San Nicola de Crissa, Cimino et Capistrano. Son voyage a abouti à un album de photographies contenant environ 540 photographies et eut aussi pour résultat un article de Carlo Levi, publié dans le numéro de mars 1952 du Courrier de l'UNESCO,${ }^{4}$ accompagné par plusieurs photographies de Seymour et des légendes détaillées.

Après la Seconde Guerre mondiale, le matériel de promotion émis par les agences humanitaires comprenait de nombreuses photographies de la reconstruction de l'Europe et des initiatives éducatives s'y rapportant. En raison de leur « plasticité médiatique », des images photographiques ont été inclues et publiées dans diverses campagnes médiatiques et dans le cadre de différents médias pour susciter un débat, former l'opinion, et définir dans la sphère publique les sujets d'importance. ${ }^{5}$ La photographie a joué un rôle clé dans ce processus, car sa force découle de sa capacité à donner un visage humain aux problèmes et aux évènements et à capter et rendre visibles des moments spécifiques qui, autrement, se perdraient dans le flux du temps. ${ }^{6}$ En outre, les dimensions technologiques de la photographie ont facilité la production institutionnelle et la gestion de la visibilité et des connaissances par le biais de la reproduction. Jay David Bolter et Richard Grusin nomment « la représentation d'un média dans un autre remédiation », et des images photographiques commandées par des agences humanitaires sont effectivement (ré)apparues - et souvent modifiées - dans de nombreux contextes et médias. ${ }^{7}$

Courrier de l'UNESCO 2, n ${ }^{\circ} 1$ (1949), p. 1, 5-9 ; Enfants d'Europe : photographies de David Seymour. Paris : UNESCO 1949 (Publication $n^{\circ}$ 403) ; Thérèse Brosse : Enfants sans foyer. Paris : UNESCO 1950 (Publication $\mathrm{n}^{\circ}$ 573).

3 Sur la collaboration entre David Seymour et Carlo Levi, voir aussi l'essai de Carole Naggar dans ce volume.

4 Carlo Levi ; L'Italie du sud mène la bataille contre l'analphabétisme. In : Le Courrier de l'UNESCO 5, $\mathrm{n}^{\circ} 3$ (1952), p. 3-5.

5 Le terme " plasticité médiatique » a été employé par Robert Hariman et John Louis Lucaites pour décrire un trait important de la photographie qui influence son rôle en tant qu'image publique, voir Robert Hariman/John Louis Lucaites : The Public Image : Photography and Civic Spectatorship. Chicago, IL : University of Chicago Press 2016, p. 13 ; voir aussi Lilie Chouliaraki/Tijana Stolic : Rethinking Media Responsibility in the Refugee «Crisis » : A Visual Typology of European News. In : Media, Culture \& Society 39, $\mathrm{n}^{\circ} 8$ (2017), p. 1162-1177 ; Ariella Azoulay : The Civil Contract of Photography. New York : Zone Books 2008.

6 John Berger : About Looking. Londres : Writers and Readers Publishing Cooperative 1980 ; Stuart Franklin : The Documentary Impulse. Londres et New York : Phaidon Press 2016.

7 Jay David Bolter/Richard Grusin : Remediation : Understanding New Media. Cambridge, MA : MIT Press 1999, p. 45. 
La publication des photographies de Seymour par l'UNESCO a été très sélective - douze photographies seulement sur un total d'environ 540 ont été publiées dans Le Courrier de l'UNESCO. L'UNESCO n'a évidemment pas souhaité publier un énorme reportage. Seymour travaillait généralement avec un très petit budget et attendait de la coopérative Magnum Photos qu'elle continue de promouvoir les images sur différents supports en diffusant en même temps un texte de Carlo Levi. ${ }^{8}$

\section{L'album « L'Italie mène la bataille contre l'anal- phabétisme » en tant qu'outil rédactionnel}

Avant d'analyser plus en profondeur le mode de narration visuelle de Seymour, je voudrais examiner de plus près l'album en tant qu'objet et les traces matérielles qu'il exhibe. ${ }^{9}$ L'album des planches-contact de Seymour n'est ni un album officiel, ni un album personnel ; il semble plutôt être un outil de travail créé par des professionnels pour les campagnes médiatiques. ${ }^{10}$

L'album débute par une légende qui lui sert également d'introduction générale et souligne son objectif principal, la lutte contre l'analphabétisme en Calabre. Il incorpore également des copies au papier carbone où sont dactylographiés des commentaires numérotés à propos des photographies des planches-contact d'une trentaine de films de moyen format ; la plupart des films comptent 12 négatifs (soit

8 Carlo Levi a publié plusieurs articles de journaux sur les problèmes politiques et sociaux en Calabre. Certains ont parus dans le New York Times sous le titre Peasants Stir in Groping Italy. In : New York Times (14 septembre 1947) ; For Freedom We Must Conquer Fear. In : New York Times (3 octobre 1948) ; Eboli Revisited: New Life Stirs. In : New York Times (13 mars 1949) ; Italy Fights the Battle of Illiteracy. In : New York Times (6 novembre 1949) ; et Italy's Peasants Look at Land Reform. In : New York Times (17 mai 1953). Pour plus d'informations sur la bataille de Levi et Seymour pour attirer l'attention des médias sur la situation en Calabre, voir l'essai de Carole Naggar dans ce volume.

9 Pour plus d'informations sur la qualité spécifique des albums historiques en tant qu'objets visuels et l'analyse des images photographiques en tant qu'objets enchevêtrés et sociaux, voir AnneMarie Garat : Photos de familles. Paris : Editions du Seuil 1994 ; Martha Langford : Suspended Conversations: The Afterlife of Memory in Photographic Albums. Montreal et Kingston : McGill-Queens University Press 2001 ; Elizabeth Edwards : Entangled Documents : Visualized Histories. In : Kristen Lubben (éd.) : Susan Meiselas : In History. New York : International Center for Photography et Göttingen : Steidl Publishers 2008, p. 330-341; Elizabeth Edwards : Photographs : Material Form and the Dynamic Archive. In : Costanza Caraffa (éd.) : Photo Archives and the Photographic Memory of Art History. Berlin : Deutscher Kunstverlag 2011, p. 47-56.

10 L'album est un classeur à anneaux identique à d'autres utilisés par l'UNESCO pour assembler les planches-contact reçues des photographes. Il est très probable que David Seymour a transmis les commentaires, les légendes et les planches-contact à l'UNESCO où ils ont été placés dans le classeur. Les archives de Magnum à New York contiennent un album noir - ce type d'album était utilisé pour tous les photographes - avec le même contenu. 
un total de 352 photographies au moyen format). La numérotation des commentaires au carbone ne semble pas suivre l'ordre chronologique de production des images qu'indique la numérotation consécutive des négatifs. En compilant l'album et en ajoutant des commentaires, David Seymour a pris des décisions précises qui lui permettraient de créer plusieurs séquences et récits interconnectés à partir de l'ensemble des photographies. ${ }^{11}$ Bien qu'il ait respecté l'ordre chronologique des photographies, son souci principal n'a pas été une chronologie linéaire de son exploration visuelle du sud de l'Italie. Au lieu de cela, il a construit des scénarios spécifiques et sélectionné des photographies spécifiques pour accompagner et renforcer ces scénarios. En sus des planches-contacts au moyen format de la première partie de l'album, des couches supplémentaires de temps et de contenu sont apportées par un très grand nombre de négatifs de petit format (réalisés par Seymour au Leica) qui constituent la seconde partie de l'album. Ces planches-contact de films de petit format suivent un ordre consécutif (L. 1 à L. 8) et comprennent de nombreux duplicata des images au moyen format, ainsi que des photographies plus spontanées de la vie quotidienne. Dans cette section, cependant, l'album propose moins de commentaires et de légendes. En bref, l'album démontre que son compilateur, David Seymour, ne voulait pas uniquement indiquer comment monter des histoires à partir des photographies, mais qu'il désirait mettre en valeur des aspects précis de ces histoires en ajoutant des légendes à une sélection de photos pour permettre aux rédacteurs de construire des histoires entièrement fidèles au récit qu'il souhaitait. Dans ce but, il a pris certaines décisions que révèlent les traces rédactionnelles à la surface de l'album. Ces traces révèlent comment Seymour pensait que l'album devrait fonctionner et déterminent le mode de perception des photographies par leurs rédacteurs et leurs lecteurs.

L'album a donc non seulement servi d'outil rédactionnel, mais également d'outil de communication entre le photographe et ses rédacteurs, ainsi qu'entre le photographe, le rédacteur et le public. ${ }^{12}$

11 Je souhaite remercier Carole Naggar pour avoir suggéré des indices qui ont permis d'identifier David Seymour comme auteur de ces commentaires et légendes. La preuve que Seymour est l'auteur est fournie par un défaut de frappe dans sa machine à écrire, et plus encore par le style, qui est identique à celui de ses autres reportages des années 1950 (y compris les erreurs d'un homme dont l'anglais n'était pas la langue maternelle).

12 Il convient de noter que l'album contient aussi plusieurs commentaires au crayon qui ont très probablement été écrits par les rédacteurs de Seymour. 


\section{Feuilleter l'album : le photographe en conversa- tion avec ses rédacteurs}

Résultat de la commande de l'UNESCO à Seymour, l'album « L'Italie mène la bataille contre l'analphabétisme » démontre que Seymour a abordé la mission de l'UNESCO dans une perspective plus large qu'une commande: il a agi en observateur sensible et respectueux et non content de nous donner des images merveilleuses des gens, de la vie quotidienne et des paysages de Calabre, il a commenté par le biais de ses photographies des questions telles que l'éducation et les activités d'apprentissage, le rôle des femmes, le manque d'infrastructures et la bataille des paysans contre la pauvreté.

L'histoire de l'album débute en première page avec la légende d'une photographie au moyen format ( $\left.\mathrm{n}^{\circ} 2\right)$ de Roggiano Gravina, un village que Seymour décrit comme faisant partie d'un « district agricole très pauvre ». Comme je l'ai mentionné ci-dessus, la longue légende sert aussi de texte d'introduction, et Seymour a noté que la fréquentation scolaire des enfants dans le village avait récemment atteint cent pour cent et que sept cents adultes suivaient des cours du soir. Selon Seymour, ces résultats ont été réalisés grâce à l'Unione per la Lotta contro l'Analfabetismo (Ligue pour la lutte contre l'analphabétisme). La légende mentionne d'autres réussites de la Ligue et les centres culturels qu'elle a mis en place :

$\mathrm{Au}$ cours des quatre dernières années, le pourcentage d'illettrés [sic] est tombé de 68 à 22 pour cent. Cette action est supervisée par le Centre de culture du peuple sous la direction de Giuseppe Zanfini, qui assume en même temps la charge d'Inspecteur régional des syndicats de Calabre. Le Centre s'est vraiment inscrit au cœur de la vie du village. Son mode de gestion est intéressant : il donne aux plus jeunes la possibilité de travailler dans un atelier rudimentaire où ils assemblent le matériel éducatif, et où on enseigne la taille du bois et des données de base de menuiserie. Les femmes y apprennent les travaux d'artisanat et la broderie, et leurs heures d'école du soir sont consacrées à la couture et aux leçons.

Seymour souligne également les tensions politiques dans le sud de l'Italie et attire l'attention sur un aspect important qui n'a pas été spécifiquement souligné dans l'article de Carlo Levi pour Le Courrier de l'UNESCO:

Comme toute autre ville ou village en Italie, Rogiano [sic] Gravina est amèrement divisé par des luttes politiques. Mais le Centre de culture du peuple a réussi à maintenir un statut apolitique. Communistes et démocrates-chrétiens sont assis côte à côte sur les bancs et font de gros efforts pour apprendre. La misère est celle de tous, et quand la vague d'occupation des terres a récemment submergé l'Italie du sud, Rogiano [sic] Gravina a offert l'extraordinaire spectacle d'un défilé réunissant les communistes et leur drapeau rouge et les démocrates-chrétiens et leur drapeau blanc : ils allaient occuper ensemble la terre en friche du Baron Campagna, un propriétaire local possédant plus de 5000 hectares. Après de dures années de bataille contre l'analphabétisme, Rogiano [sic] Gravina nourrit de nouveaux espoirs. Le Gouvernement construit une grande école élémentaire qui va remplacer les 42 salles de classes privées dispersées 
dans le village. Un lopin de terre appartenant au village a été récemment attribué au Centre, qui a le projet d'y construire un nouveau bâtiment comprenant des salles de classes et des ateliers pour adultes. Mais ils ont besoin de fonds, et ils espèrent que l'UNESCO va les aider.

Sur la première double page suivante de l'album, à la planche contact 50-3-9 sur la page de droite, Seymour a attiré l'attention sur plusieurs photographies en leur attribuant des légendes sur la page de gauche. ${ }^{13}$ La première photographie $\left(n^{0} 4\right)$ montre l'emplacement où doit être construit le nouveau centre et la seconde image $\left(n^{\circ} 6\right)$ représente un " paysan âgé » prenant une leçon d'écriture. De plus, il y a une remarque rédactionnelle sur le papier carbone concernant l'éducation des adultes, faisant référence à une séquence d'images sur cette première planche-contact de l'album qui montre des hommes âgés qui assistent à une leçon d'écriture après le travail. Sur la double page suivante, les remarques sur une coupure au papier carbone font référence à plusieurs photographies des planches-contact 50-3-9 et 503-10, qui montrent les mains d'un homme adulte tenant une plume pour la première fois. Seymour a clairement désigné trois photographies : la photo $\mathrm{n}^{0} 5$ de la planche contact 50-3-9 et les photos $\mathrm{n}^{\text {os }} 15$ et 21 de la planche-contact 50-3-10. Celles-ci sont en effet des photographies très fortes et ont été publiées dans l'article du Courrier de l'UNESCO, et la photographie $\mathrm{n}^{\circ} 15$ figure même en couverture du magazine.

Sur la troisième planche-contact de l'album (50-3-11), Seymour a choisi les photographies $\mathrm{n}^{\text {os }} 27,28$ et 33, qui montrent des adolescents et des jeunes gens scolarisés. La quatrième planche-contact (50-3-12) est accompagnée d'un commentaire plus long sur le papier carbone, reprenant certaines informations données précédemment et ajoutant que les paysans et leurs enfants devaient faire de longues distances à pied pour aller à l'école. Il est également mentionné que " La méthode pédagogique est intéressante : un bon nombre de soirées sont consacrées à la culture générale, avec des périodes de questions et réponses » et que « les jeunes gens du village [...] fabriquent des fournitures pédagogiques telles que des cartes de géographie, des meubles pour les salles de classe, de l'équipement de laboratoire de base, etc. " Nous apprenons également la création d'un groupe de musique et d'un groupe de théâtre amateur, et on nous dit que les enseignants étaient très respectés et donnaient régulièrement des conseils juridiques pour aider les paysans à traiter avec les autorités officielles, et que des médecins donnaient des consultations dans les centres culturels. La cinquième planche-contact (50-3-13) est accompagnée d'une observation sur le taux élevé d'analphabétisme chez les femmes qui apprenaient parfois à lire et à écrire en brodant des lettres. Une autre remarque a trait au « système de lettres mobiles » utilisé dans les salles de classe. Seymour a également

13 Les planches-contact indiquent des références internes de Magnum : l'année où les photos ont été prises (1950), le nombre de reportages faits jusque-là par Seymour dans l'année (3) et le numéro consécutif du nombre total de planches-contact figurant dans les archives de Magnum ( $9 \mathrm{ff}$.). 
souligné une photo ( $\mathrm{n}^{\circ}$ 60) qui montre la fabrication de matériel pédagogique. L'UNESCO a toutefois préféré publier une autre image sur le même sujet ( $\mathrm{n}^{\circ} 37$ sur la planche-contact précédente).

Sur la sixième planche-contact (50-3-14), qui ne comporte pas de commentaires, Seymour a sélectionné trois photographies pour accentuer des aspects spécifiques de son histoire: une photographie $\left(\mathrm{n}^{\circ}\right.$ 64) montre une vue générale du village et a été publiée inversée dans Le Courrier de l'UNESCO ; la photographie suivante $\left(n^{\circ} 68\right)$ montre une scène de rue à Roggiano Gravina ; et la troisième photo $\left(n^{\circ} 70\right)$ est un gros plan de la même scène de rue, centré sur un groupe de femmes et de jeunes filles ; selon la description de Seymour, l'une d'elles est en train de faire ses devoirs.

Trois commentaires sur la brièveté de la scolarisation obligatoire ont été ajoutés à la septième planche-contact (50-3-15) pour souligner l'importance de l'éducation des adultes, des clubs de jeunes, de la formation professionnelle continue, de la création de bibliothèques et de l'organisation de manifestations culturelles.

La huitième planche-contact (50-3-16) comporte un grand nombre de photos de femmes portant de lourds fardeaux sur leurs têtes. Seymour n'a pas commenté ce thème à ce stade mais a plutôt mis en avant une photographie $\left(n^{\circ} 83\right)$ qui représente l'entrée du Centre culturel de Roggiano et qui a ensuite été sélectionnée pour publication par l'UNESCO.

La neuvième planche-contact (50-3-17) est accompagnée de deux commentaires sur la page d'en face qui mettent l'accent sur la photographie d'un enseignant guidant la main d'élèves adultes et d'autres photos de leçons d'écriture que suivent des hommes âgés. Quant à la dixième planche-contact (50-3-18), Seymour a souligné trois photographies ( $\mathrm{n}^{\text {os }} 109,114$ et 117) en leur ajoutant des légendes détaillées. Elles montrent le directeur du Centre culturel en train d'aider les paysans à remplir les formulaires officiels et de lire la lettre d'un fils émigré depuis longtemps à sa mère illettrée ; la légende mentionne qu'Emilia, la mère, a immédiatement demandé à être admise à l'école, « pour pouvoir à l'avenir lire toute seule la lettre de son fils. »

La planche-contact suivante (50-3-19) est accompagnée de commentaires qui font référence à l'éducation des femmes et précisent que les femmes étaient souvent accompagnées de leurs enfants lorsqu'elles allaient à l'école. Une image très forte de cette planche-contact $\left(\mathrm{n}^{\circ} 123\right)$ a été sélectionnée pour publication par l'UNESCO (mais pas par Seymour) : elle représente une femme et sa petite fille qui font ensemble un exercice de lecture.

La planche-contact 50-3-20 n'est pas accompagnée d'un commentaire, mais d'une légende détaillée pour l'une des photographies ( $\mathrm{n}^{\circ} 139$ ) expliquant le problème de la propriété foncière et la difficulté à gagner sa vie en Italie du sud. La photo montre Oreste Rossini, un père de cinq enfants, qui possédait un petit terrain et qui assistait régulièrement à des cours du soir. L'UNESCO a toutefois sélectionné pour publication une autre photographie $\left(n^{\circ} 135\right)$ de cette planche-contact, qui ne fait 
pas référence à une personne ni à des expériences spécifiques mais dépeint des hommes qui travaillent la terre.

La planche-contact suivante (50-3-21) a aussi pour sujet la propriété foncière et l'occupation des terres. Seymour a mis en valeur cinq photographies ( $n^{\text {os }} 145-149$ ) pour illustrer cette dimension spécifique de son histoire. Ces photos évoquent son reportage sur la guerre civile espagnole mais on constatera que l'UNESCO ne les a pas choisies pour les publier. Des bâtiments scolaires et une femme portant un fardeau sont les sujets des photographies choisies ensuite ( $n^{\text {os }} 158,161$ et 168) sur la planche-contact 50-3-22. La légende de la photographie $\mathrm{n}^{\mathrm{o}} 168$ - une femme portant un fardeau - témoigne du désir de Seymour de raconter une histoire et de conseiller le rédacteur. On peut lire :

Une des nombreuses photos de femmes portant divers fardeaux sur leur tête. J'ai l'idée que l'on pourrait utiliser ces photos comme introduction symbolique à l'histoire. Je n'ai pas encore réussi à trouver la formule exacte, mais l'idée générale c'est que les gens de Calabre se sont jusqu'ici servis de leur tête [sic] pour porter des fardeaux, et parfois de très lourds fardeaux, mais découvrent maintenant qu'ils peuvent se servir de leur tête pour leur éducation.

Les commentaires de la quinzième planche-contact (50-3-23) mentionnent les plaintes de l'institutrice Olga Natale sur l'infrastructure médiocre de l'école et la difficulté à trouver une salle de classe adéquate dans un village du nom de Cimino. Ici, Seymour sélectionne quatre photographies comme éléments importants de son histoire. La légende de l'une de ces photos ( $\left.n^{\circ} 72\right)$ est plus ou moins identique au commentaire général en haut de la page et souligne la piètre construction et l'équipement rudimentaire des salles de classe. La porte ouverte de la salle de classe attire l'attention du lecteur sur un jeune garçon qui garde ses moutons - un thème qui relie cette photo à trois autres photos sélectionnées sur cette planche-contact ( $\left.\mathrm{n}^{\text {os }} 178-180\right)$, des photographies romantiques d'un jeune berger qui suggèrent aussi le thème du travail des enfants. La seizième planche-contact (50-3-24) comprend d'autres photographies de la salle de classe et du jeune berger de Cimino et elle est accompagnée d'un bref commentaire. Il est intéressant de noter qu'une photographie ( $\left.n^{\circ} 190\right)$ de cette planche-contact (sa légende fait référence au travail des enfants) a été sélectionnée par Seymour plus avant dans l'album, en liaison avec une séquence sur l'enseignant Antonio Janni. La dix-septième planche-contact (503-25) montre à nouveau des scènes de salle de classe - Seymour a sélectionné la photo $\mathrm{n}^{\circ} 193$ - et un portrait saisissant d'un jeune garçon ( ${ }^{\circ}$ 97) que l’UNESCO a publié.

La dix-huitième planche-contact (50-3-26) montre des images d'une femme âgée au visage très frappant et de deux petites filles probablement sur le chemin de l'école à côté de photographies de cours d'éducation pour adultes. Seymour a souligné une photographie chaleureuse $\left(\mathrm{n}^{\circ} 210\right)$ d'un père qui fait des exercices d'écriture assis près de son fils, plein de vie et de curiosité. 
La double page suivante, à la planche-contact 50-3-27, regroupe les légendes de trois photographies ( $\mathrm{n}^{\text {os }} 219,224$ et 226). Sur la page de gauche, nous voyons des marques supplémentaires de rédaction. Une des légendes (pour la photographie $\mathrm{n}^{\circ}$ 226) présente Antonio Janni, un enseignant qui avait perdu sa citoyenneté américaine parce qu'il s'était battu dans les rangs de l'armée italienne pendant la guerre, et qui à présent, devait parcourir seize kilomètres par jour pour arriver aux écoles du village. La planche-contact suivante (50-3-28) est également consacrée à Antonio Janni et à son rôle dans les salles de classe et hors de l'école. Pour relier les deux commentaires à propos d'Antonio Janni et des bâtiments scolaires rudimentaires, Seymour a mis l'accent sur trois photographies ( $n^{\text {os }} 190,191$ et 236), dont deux figuraient sur une planche-contact précédente. La photo $\mathrm{n}^{\circ} 239$, qui montre Janni debout au milieu de la salle de classe, n'avait pas été choisie par Seymour mais a été sélectionnée par l'UNESCO pour être publiée.

La vingt-et-unième planche-contact (50-3-29) se concentre sur le paysage et les longues distances que jeunes et moins jeunes doivent couvrir pour arriver jusqu'aux salles de classe. Un commentaire informe les rédacteurs que les chemins étaient très dangereux en hiver. La double page suivante (50-3-30) répète cette information et inclut aussi des légendes pour quatre photographies sélectionnées ( ${ }^{\text {os }} 262$, 256, 259 et 299), dont l'une ( $n^{\circ} 299$ ) figure sur une planche-contact qu'on retrouve plus tard dans l'album. La photographie $\mathrm{n}^{\circ} 265$, qui dépeint le modeste intérieur d'une maison paysanne, a été sélectionnée pour publication par l'UNESCO. La planche-contact suivante (50-3-31) montre les maisons et les familles sans autre commentaire. La légende de l'une des photographies choisies sur cette planche-contact $\left(\mathrm{n}^{\circ}\right.$ 276) est placée sur la page de gauche de la double page suivante («Vue de Saucci avec les nuages recouvrant les pics montagneux »), où Seymour a ajouté deux autres légendes pour les photographies figurant sur la planche-contact 50-332 : une photographie $\left(n^{\circ} 278\right)$ des enfants poussant le scooter de leur instituteur (Antonio Janni) et une ( $n^{\circ}$ 61) des enfants qui marchent dans une région montagneuse. Mais il y a aussi un autre commentaire se rapportant à Antonio Janni : « C'est sa première année d'enseignement à l'école de Saucci. Il a hérité du travail de son cousin, qui a contracté une pneumonie et qui est mort, probablement d'épuisement. Ce n'est pas étonnant, car le travail est extrêmement dur. " Le reste du commentaire répète l'information donnée dans une légende précédente qui décrivait pour la première fois l'instituteur.

La double page suivante (qui inclut la planche contact 50-3-33) montre des habitations misérables - Seymour a souligné la photographie $n^{\circ} 309$ sur une planche-contact ultérieure (50-3-38) pour montrer le mauvais état des habitations paysannes - et plusieurs photos d'un jeune garçon tenant un cheval en bride. Le commentaire indique que l'un des plus gros problèmes liés à l'analphabétisme est le travail des enfants. Sur la planche-contact 50-3-34, Seymour a mis l'accent sur trois photographies ( $\mathrm{n}^{\text {os }} 314,324$ et 319). Les légendes font référence aux femmes et aux enfants « qui utilisent leurs têtes » pour porter des fardeaux et à une famille qui 
travaille dans les champs. La planche-contact 50-3-35 et deux des trois photographies soulignées ( $\mathrm{n}^{\circ} 328$ et 330 ) font à nouveau référence aux femmes qui portent des fardeaux - l'un des sujets préférés de Seymour. L'UNESCO a choisi de publier la photographie $n^{\circ} 330$ et a donc suivi le scénario de Seymour, mais leur légende est moins provocante que les commentaires de Seymour. Une troisième photo soulignée $\left(n^{\circ} 334\right)$ montre, pour une fois, une photographie tout à fait idyllique : " Jeunes bergers sur la route de Spezzano Albanese ». Seymour a souligné sur la planche contact 50-3-36 trois photographies ( $n^{\text {os }} 345,346$ et 338) qui représentent à nouveau un jeune berger et Oreste Rossini assistant à un cours du soir. Les deux commentaires faisant référence à cette planche-contact expliquent que les jeunes garçons ne peuvent pas aller à l'école parce qu'ils doivent garder les troupeaux de moutons de leur famille. La photographie $n^{\circ} 38$ où Oreste Rossini figure à l'arrièreplan a été publiée par l'UNESCO.

La planche-contact suivante (50-3-37) montre le paysage de Calabre, et Seymour y a sélectionné toutes les photographies qui donnent un contexte visuel important à son histoire. La planche-contact 50-3-38 est accompagnée de deux commentaires à propos du manque de routes qui rendent la région difficile d'accès, et il est à noter que cette dernière planche-contact au moyen format est la seule à interrompre la numérotation consécutive des négatifs.

La deuxième partie de l'album est constituée de planches-contacts de films de petit format qui montrent des photographies de la vie quotidienne - il semblerait que Seymour ait profité de la souplesse d'emploi de son Leica, un appareil photo plus petit. Dans cette partie, il a sélectionné moins de photographies pour accompagner son histoire - une des explications pourrait être que beaucoup de photos étaient des duplicatas de photographies au moyen format. L'UNESCO a sélectionné une seule photo de petit format ( $\mathrm{n}^{\circ} 8$ sur la cinquième planche-contact au Leica). C'est une scène de rue avec deux garçons et un âne, et une voiture à l'arrière-plan.

En feuilletant l'album, on découvre que sa fabrication a joué un rôle clé dans l'élaboration et la création de récits attrayants sur la bataille contre l'analphabétisme en Italie du sud. Ce n'est donc ni la chronologie ni le facteur d'authenticité de la photographie, mais plutôt les rencontres et les expériences du photographe avec des personnes véritables qui ajoutent de nouvelles perspectives à l'histoire. Seymour a capté visuellement la vie des personnes et leur environnement. Il a mis l'accent sur leur importance historique en compilant l'album et a ainsi raconté des histoires uniques et révélatrices qui, sans lui, seraient tombées dans l'oubli. 


\section{Conclusion : La photographie en tant que techno- logie médiatrice des causes humanitaires}

Je pense que nous pouvons affirmer sans crainte que cet album a fonctionné comme un outil rédactionnel et un moyen de communication entre le photographe et ses rédacteurs. Cependant, ce n'est pas Seymour mais ses rédacteurs qui ont finalement décidé quels seraient les éléments narratifs à souligner, qui deviendrait une figure publiquement reconnue avec un nom et un visage, et quelles photographies seraient utilisées pour déclencher un débat public et lui donner forme. Lors de la compilation de l'album, Seymour avait l'idée de raconter différentes histoires sans prendre en compte la chronologie de ses prises de vue. À travers ses légendes et ses commentaires, il a créé des scénarios qui se recoupaient et fonctionnaient en tant qu'éléments flexibles de la même boîte à outils : l'album. Quelques-uns de ces scénarios sont les suivants : (1) l'occupation des terres par les paysans pour combattre la pauvreté, avec comme protagoniste principal Oreste Rossini, qui a aussi été un participant enthousiaste des cours du soir ; (2) le manque d'infrastructures, documenté par des photographies de bâtiments scolaires rudimentaires, un matériel pédagogique fait main et le manque de routes ; (3) l'histoire de l'instituteur Antonio Janni et son rôle dans la communauté ; (4) le manque général de stimulation intellectuelle et de possibilités d'éducation pour les filles et les femmes, symbolisé par les lourds fardeaux qu'elles portaient sur leurs têtes ; et, enfin, (5) le problème du travail des enfants.

En conclusion, jetons un coup d'œil à l'article de Carlo Levi dans le numéro de mars 1952 du Courrier de l'UNESCO pour voir comment la cause de la bataille contre l'analphabétisme en Italie du sud a été (différemment) médiatisée par la rédaction institutionnelle. ${ }^{14}$ Levi mentionne que les statistiques les plus récentes sur l'analphabétisme en Italie du sud datent de 1931 et ne sont donc pas vraiment à jour. Puis, poursuit en esquissant une liste des causes et problèmes liés à l'analphabétisme - absence de structures politiques démocratiques dans cette région, manque de terres cultivées, mauvaises conditions sanitaires, prévalence du paludisme, manque d'entreprises et d'industries et, enfin, manque d'accès de la population aux médias et médiocrité des infrastructures publiques des villages. Levi insiste également sur la richesse de la culture paysanne fondée sur les traditions orales, alors que l'écriture, selon l'expression de Levi, « est pour les paysans un symbole de l'éloignement du gouvernement central, d'un lien féodal qui n'a jamais évolué en

14 En fait, l'article publié par Le Courrier de l'UNESCO était identique à celui que Levi avait déjà publié dans le New York Times, voir Levi : Italy Fights the Battle of Illiteracy. Toutefois, l'article du New York Times était illustré de cinq photographies de John Swope (1908-1979), qui travaillait aussi pour Life Magazine. 
droit de citoyenneté à part entière $" .^{15}$ Tout en mettant l'accent sur le manque de bâtiments scolaires et de matériel pédagogique et de publications pour les enfants et les adultes, Levi souligne aussi l'immense enthousiasme des résidents pour une éducation qui leur permettrait d'améliorer leur situation et de devenir des citoyens respectés. Il conclut en décrivant le succès du travail de la Ligue nationale pour la lutte contre l'analphabétisme et des « comités locaux de villages pour combattre l'analphabétisme $"{ }^{16}$ Les onze photographies qui illustrent l'article sont accompagnées de courts résumés des commentaires et des légendes rédigés par Seymour. La sélection de photos opérée par l'UNESCO n'a pas systématiquement suivi les observations visuelles perspicaces de Seymour et ses commentaires textuels, mais a plutôt choisi d'offrir une perspective plus neutre.

Cependant, il y a manifestement plusieurs thèmes qui se chevauchent dans les histoires de Seymour et de Levi. Alors que Seymour a travaillé comme " ami » respectueux et observateur des gens et s'est surtout attaché à documenter la vie de tous les jours, Levi a adopté une approche plus intellectuelle et analytique. Les photographies de Seymour qui accompagnent le texte de Lévi ont ainsi servi d'outils qui montrent des visages, des lieux et des activités en tant que moments vécus prolongés qui impliquent et touchent divers publics jusqu'à aujourd'hui. ${ }^{17}$

David Phillips décrit la photographie documentaire comme une " combinaison de preuves et d'instructions » qui « implique nécessairement diverses techniques rhétoriques et esthétiques qui associent réalité et sentiment, information et effet, factualité avec polémique $»{ }^{18}$ Il nous faut bien dire que cette définition ne rend pas pleinement justice au mode de fonctionnement réel de la photographie. En fait, on n'a accordé que peu d'attention à ce que fait la photographie en tant que technologie à part entière. C'est particulièrement vrai dans le domaine de la photographie humanitaire, qui invite naturellement à se concentrer sur les dimensions éthiques et affectives de son contenu. Il est donc nécessaire d'examiner la photographie humanitaire sous un angle différent et d'ouvrir de nouveaux horizons de recherche en la considérant également sous un angle différent et avec une perspective " matérielle-herméneutique » et en l'analysant comme une pratique et une technologie

15 Levi : L'Italie du sud mène une dure bataille contre l'analphabétisme.

16 Ibid., p. 5.

17 Voir Geoff Dyer : The Ongoing Moment. New York : Vintage Books 2007. Le critique d'art, poète, écrivain et peintre britannique John Berger décrit les photographies comme des objets vivants : "Les photographies sont des vestiges du passé, des traces de ce qui s'est passé. Si les vivants intégraient en eux-mêmes ce passé, si le passé devenait partie intégrante des personnes qui font leur propre histoire, alors toutes les photographies retrouveraient un contexte vivant, elles continueraient d'exister dans le temps, au lieu d'être des moments d'arrêt. » Voir John Berger : About Looking. Londres : Writers and Readers Publishing Cooperative 1980, p. 57. Pour la version française : John Berger : Voir le Voir. Paris : B42 2014.

18 David Philipps : Actuality and Affect in Documentary Photography. In : Richard Howells/Robert Matson (éds.) : Using Visual Evidence. Maidenhead : McGraw-Hill/Open University Press 2009, p. 64. 
comportant « un poids historique et moral $» .^{19}$ S’appuyant sur des études de philosophie de la technologie, cette perspective de recherche met en lumière les aspects technologiques et matériels cachés ou même effacés de l'interprétation et de la définition du sens. ${ }^{20}$ L'application de cette perspective aux albums de photographie permet d'analyser les photographies en tant que réseaux enchevêtrés de sens qui présentent, traduisent et trament le monde de manière spécifique. Cela veut dire que nous devons nous concentrer sur la « vie » physique des photographies, analyser la complexité de leurs contextes, leurs inscriptions et apparences matérielles, et étudier leur fabrication et leur traitement spécifique en tant qu'objets matériels, assemblés, reproductibles et mobiles. ${ }^{21}$ Cela implique de se concentrer non pas tant sur une « lecture symptomatique » de la photographie - extraire un sens en interprétant des images - mais d'examiner les caractéristiques tangibles des photographies c'est-à-dire la matérialité, la technologie et les diverses manifestations de la photographie. ${ }^{22}$ De fait, il faudrait que la recherche visuelle prenne note, cartographie et analyse les structures manifestes et imbriquées d'un vaste ensemble de photographies et fournisse de nouvelles connaissances sur le mode de création des causes humanitaires ou éducatives à la suite de dimensions matérielles et techniques et de processus connexes de fabrication du sens.

19 Elizabeth Edwards : Photography and the Material Performance of the Past. In : History and Theory 48 (2009), p. 130, 135.

20 Don Ihde : Expanding Hermeneutics : Visualism in Science. Evanston, IL : Northwestern University Press 1999.

21 Je me réfère surtout ici aux recherches de l'historienne de la photographie Elizabeth Edwards. Voir Elizabeth Edwards : Photographs and the Sound of History. In : Visual Anthropology 21, $\mathrm{n}^{\circ} 1$ (2005), p. 27-45; voir les notes 9 et 19 de cet essay pour d'autres ouvrages de cet auteure.

22 Stephen Best/Sharon Marcus : Surface Reading : An Introduction. In : Representations 108, $\mathrm{n}^{\circ} 1$ (2009), p. 1-21; Arun Appadurai : The Social Life of Things : Commodities in Cultural Perspective. Cambridge : Cambridge University Press 1986. 